\title{
Three-Dimensional Composition Analysis of SnAg Solder Bumps by Ultraviolet Femtosecond Laser Ablation Ionization Mass Spectrometry
}

\author{
A. Cedeño López ${ }^{\star a}$, V. Grimaudo ${ }^{b}$, A. Riedo ${ }^{c}$, M. Tulej $^{\mathrm{b}}$, R. Wiesendanger ${ }^{\mathrm{b}}$, \\ R. Lukmanov ${ }^{\mathrm{b}}$, P. Moreno-García ${ }^{\mathrm{a}}$, E. Lörtscher ${ }^{\mathrm{d}}$, P. Wurz ${ }^{\mathrm{b}}$ and P. Broekmann ${ }^{\mathrm{a}}$ \\ ${ }^{a}$ Department of Chemistry and Biochemistry, Interfacial Electrochemistry Group, University of Bern, \\ Freiestrasse 3, 3012 Bern, Switzerland. E-mail: alena.cedeno@dcb.unibe.ch; Tel: +41 316314269 \\ ${ }^{\mathrm{b}}$ Physics Institute, Space Research and Planetary Sciences, University of Bern, Sidlerstrasse 5, 3012 Bern, \\ Switzerland \\ ${ }^{\mathrm{c}}$ Laboratory for Astrophysics, Leiden Observatory, Leiden University, Niels Bohrweg 2, 2333 CA Leiden, \\ The Netherlands \\ ${ }^{\mathrm{d}}$ IBM Research Zurich, Säumerstrasse 4, 8803 Rüschlikon, Switzerland
}



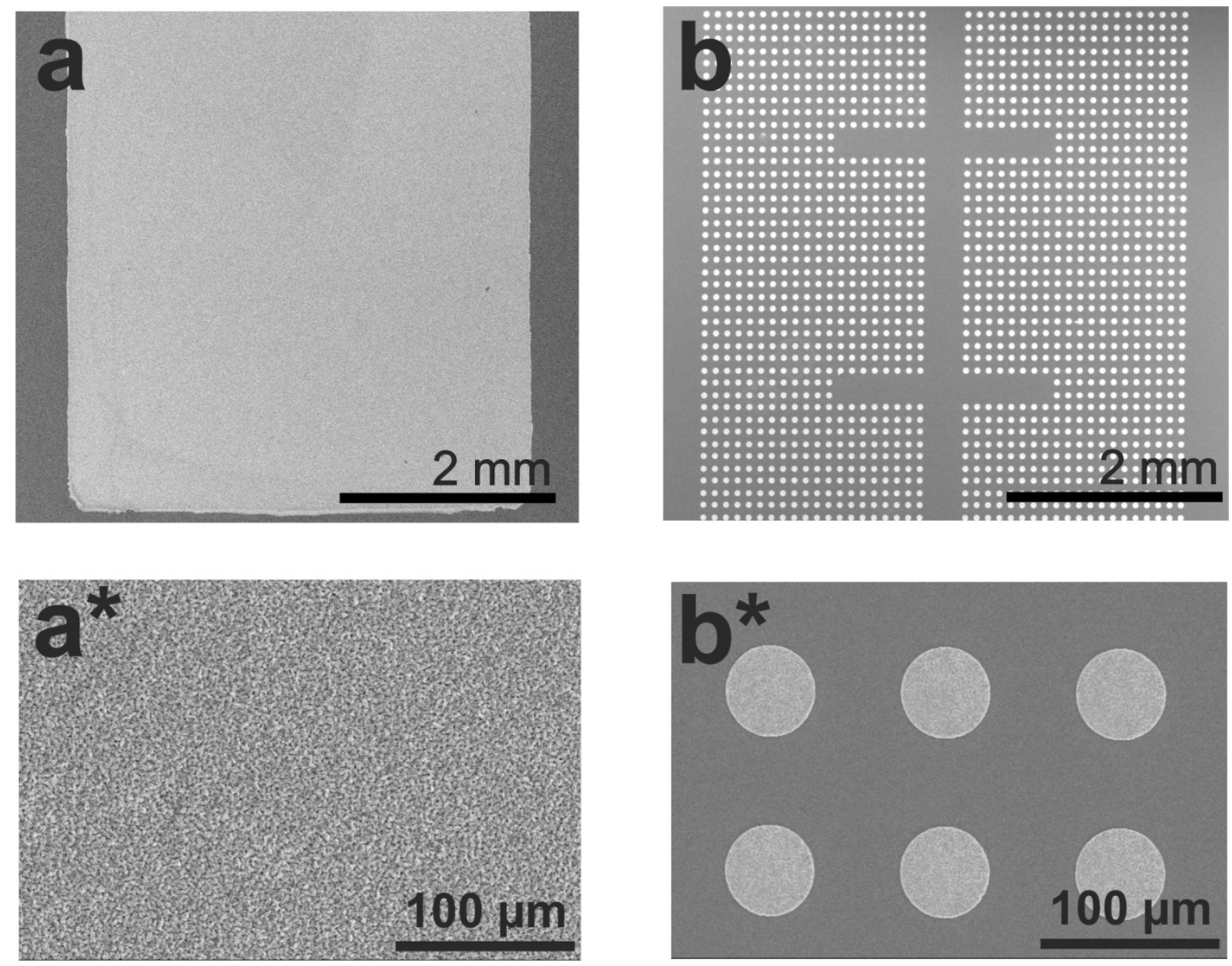

Figure S1. Top-down SEM images of SnAg film and bump electrodepositions. Panel a) and b) are overview images of the wafer die layout for SnAg film and bump plating experiments, respectively. In both cases, the surface area exposed to the plating bath is $0.25 \mathrm{~cm}^{2}$. Note that in case of the patterned wafer coupon, the stated current density has to be understood with respect to the electrochemically active surface area. In panels $a^{*}$ ) and $b^{*}$ ) a zoom-in into panels a) and b) is shown. These images illustrate the significantly higher complexity of the laterally confined bumps and their array structure on the wafer die and explain why the use of simplified model systems for screening applications is desirable. 
a

increasing layer thickness

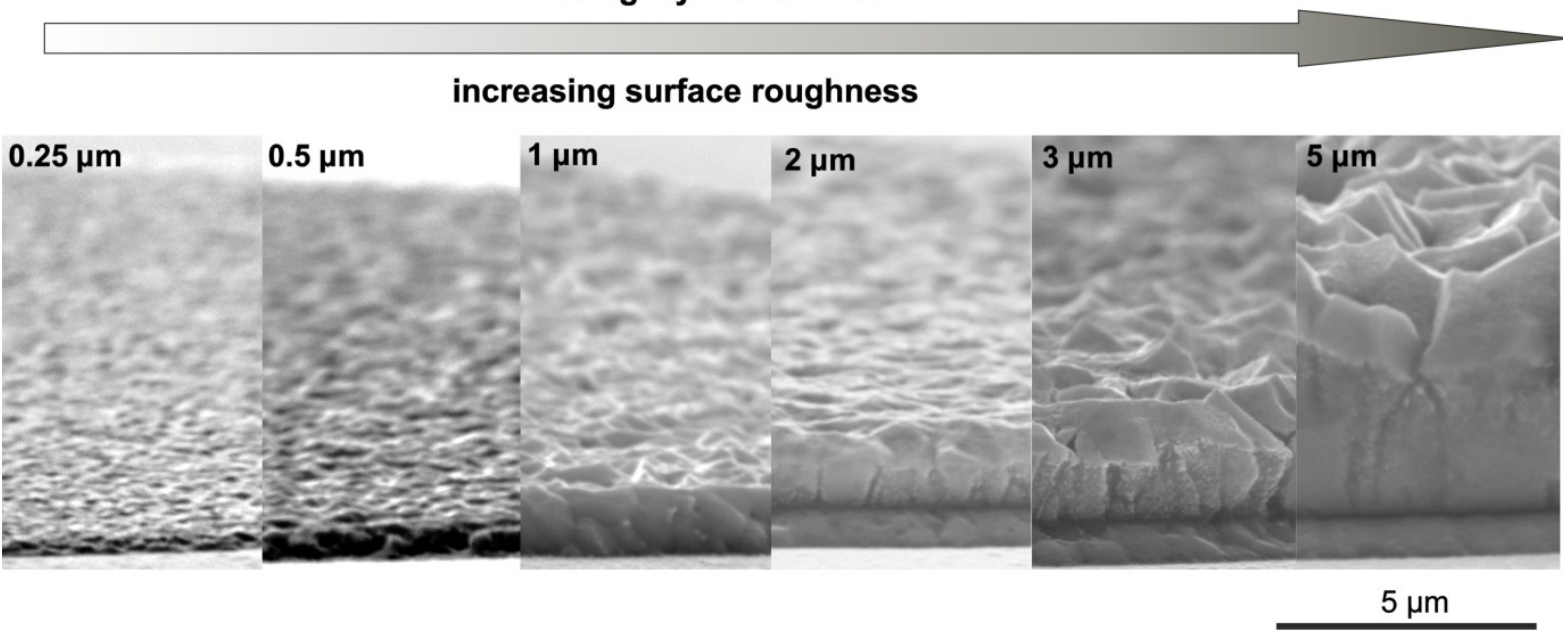

b

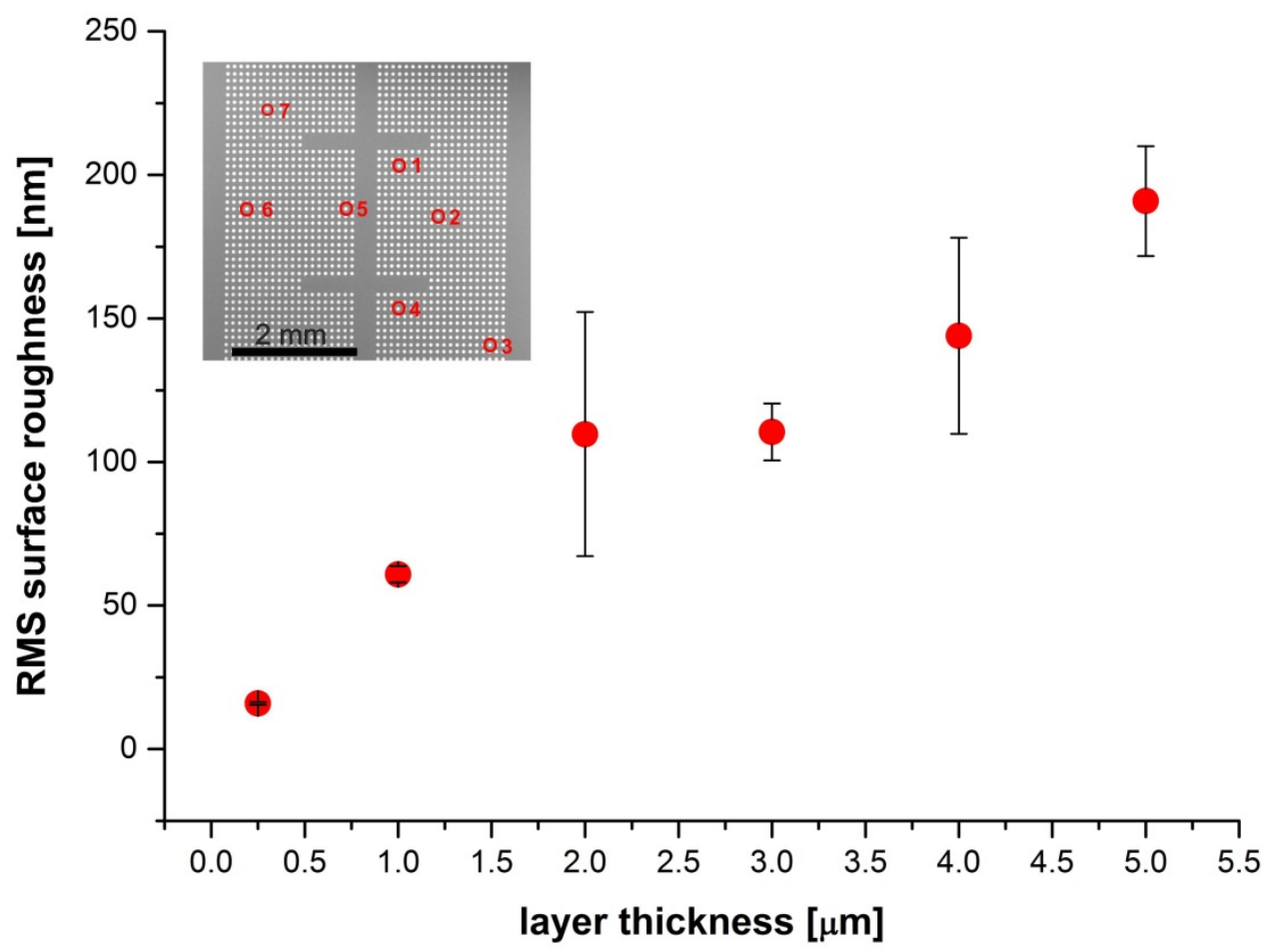

Figure S2. a) SEM side view study on the qualitative development of surface roughness with increasing layer thickness of electrodeposited SnAg bumps. Electrodepositions were carried out with Chemistry B and plating parameters 2 described in Tables 1 and 2 in the manuscript, respectively. Layer deposit thickness was varied from $0.25-5 \mu \mathrm{m}$. b) AFM-based quantitative comparison of RMS surface roughness values obtained for the SnAg alloy electrodepositions shown in panel a) (4 $\mu \mathrm{m}$ deposit thickness is additionally show in b) ). The red data points indicate the mean RMS value for central AFM scans (25x25) on 7 different SnAg bumps located at defined positions throughout the wafer die layout, which are indicated in the figure inset. 

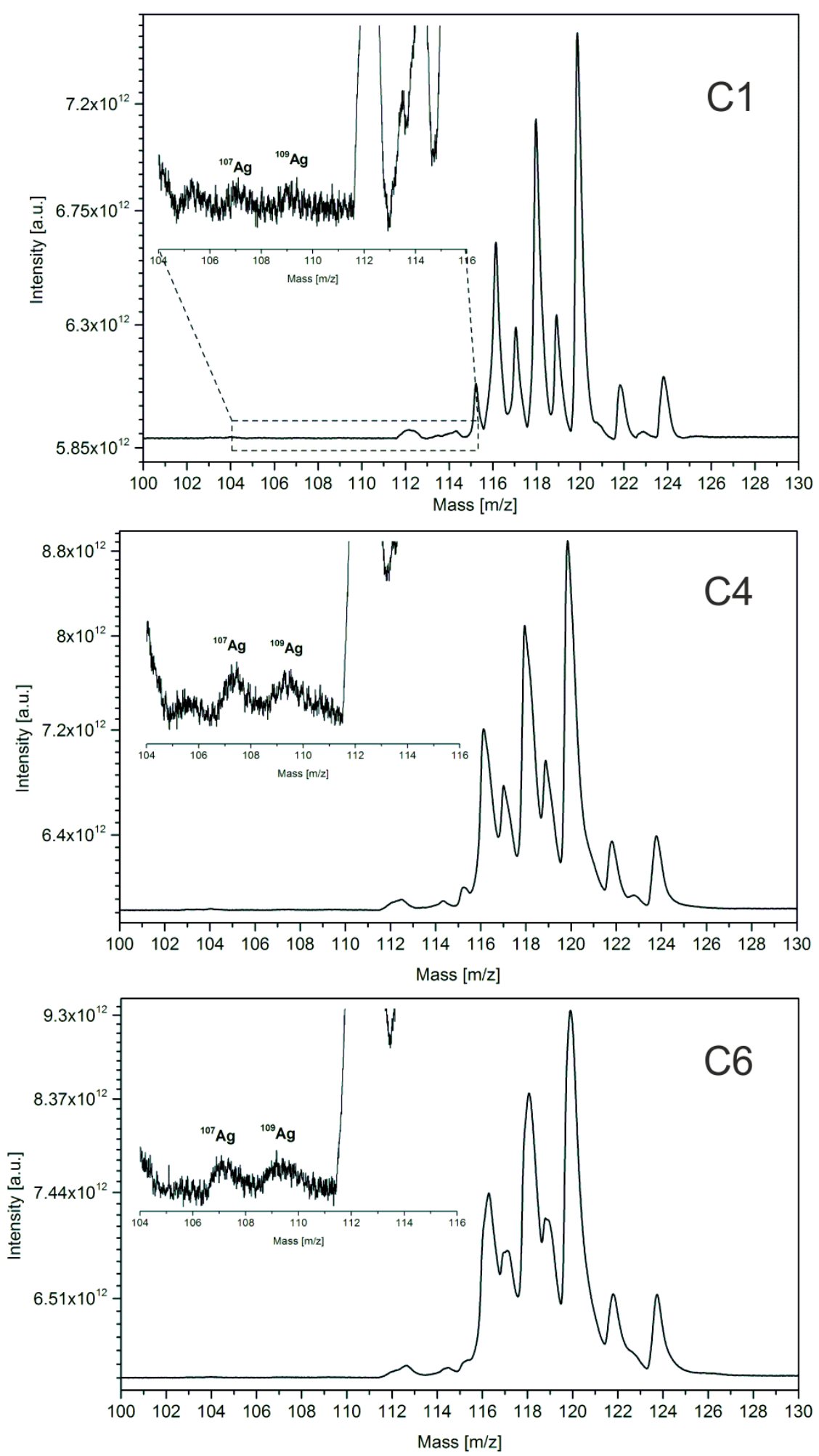

Figure S3. Excerpts of mass spectra showing the $\mathrm{m} / \mathrm{z}$ range of 100-130 obtained at different laser pulse energies (C1: $0.15 \mu \mathrm{J}$, C4: $0.33 \mu \mathrm{J}$, C6: $0.51 \mu \mathrm{J}$ ) shown in Fig. 3 for measurements on the certified reference sample BCS-CRM No. 347 (BAS Ltd., UK). 15000 single mass spectra were accumulated to build each panel. The effect of laser pulse energy on the signal-to-noise ratio and the mass resolution as discussed in the main text can clearly be seen. For the depth profiling experiments shown in Figs. 5 and 6, C4 conditions $(0.33 \mu \mathrm{J})$ were used. 


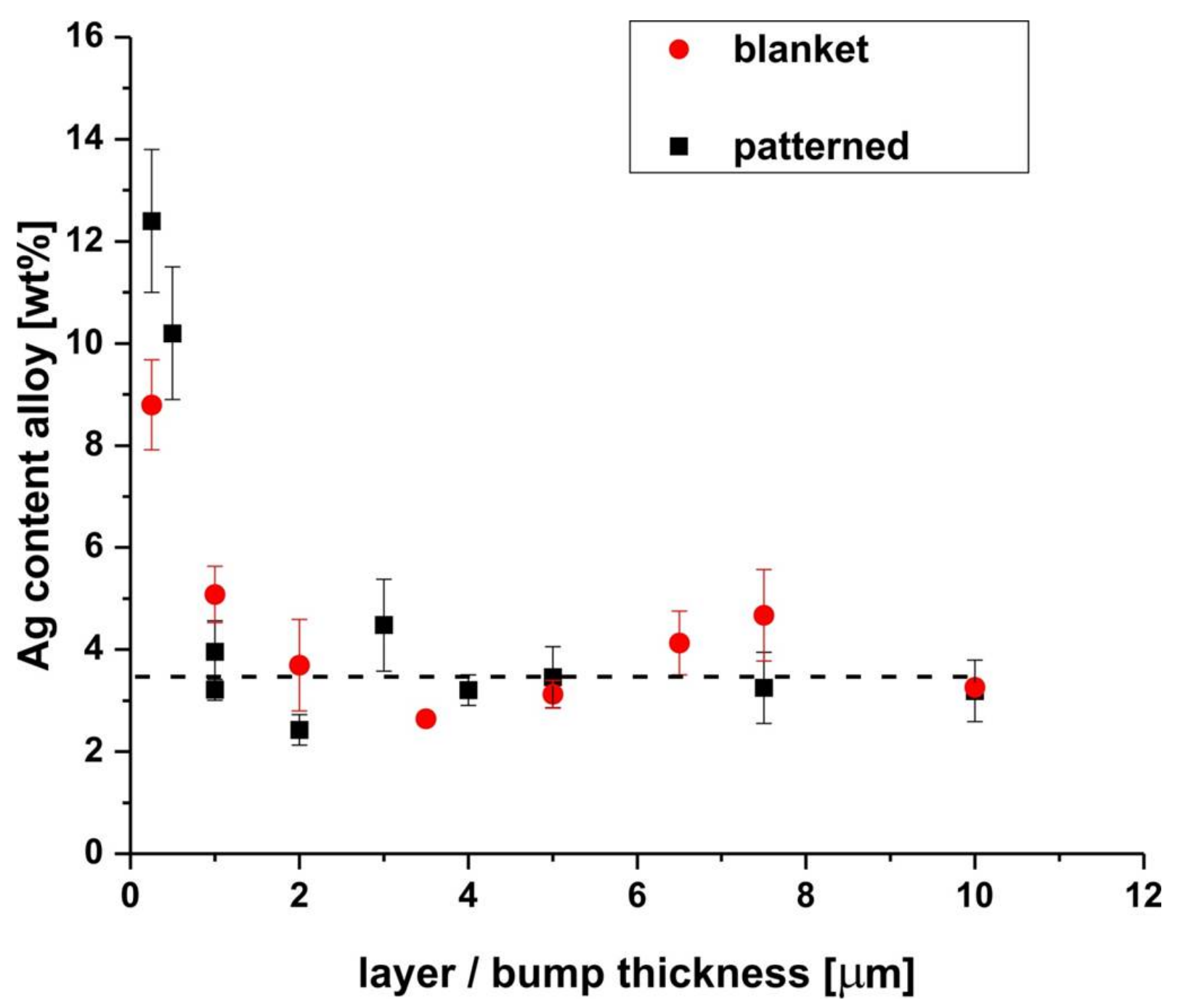

Figure S4. Dependence of the EDX-determined Ag alloy content on the electrodeposit layer thicknesses for SnAg bumps and layers. Electrodepositions were prepared with Chemistry B and plating parameter set 1 given in Tables 1 and 2 of the manuscript, respectively. The red dots represent the mean Ag alloy content determined by EDX averaged over 3 positions on two independent film or electrodeposits, the black squares stand for the mean Ag alloy content averaged over 3 positions on two independent bump electrodeposits. The development of the Ag alloy content with electrodeposit layer thickness is very similar and stabilizes around the eutectic value (indicated by the dashed line) for layer thicknesses above $1 \mu \mathrm{m}$ in both systems for a fixed plating bath composition. Hence, results obtained from EDXbased analysis of the Ag content in SnAg layer samples can be considered transferable to SnAg bumps, given that those were deposited under equivalent plating conditions and with equal plating bath composition. This is beneficial, as screening experiments are faster and significantly more inexpensive on blanket wafer coupons than on patterned wafer coupons (compare Fig. S1). 


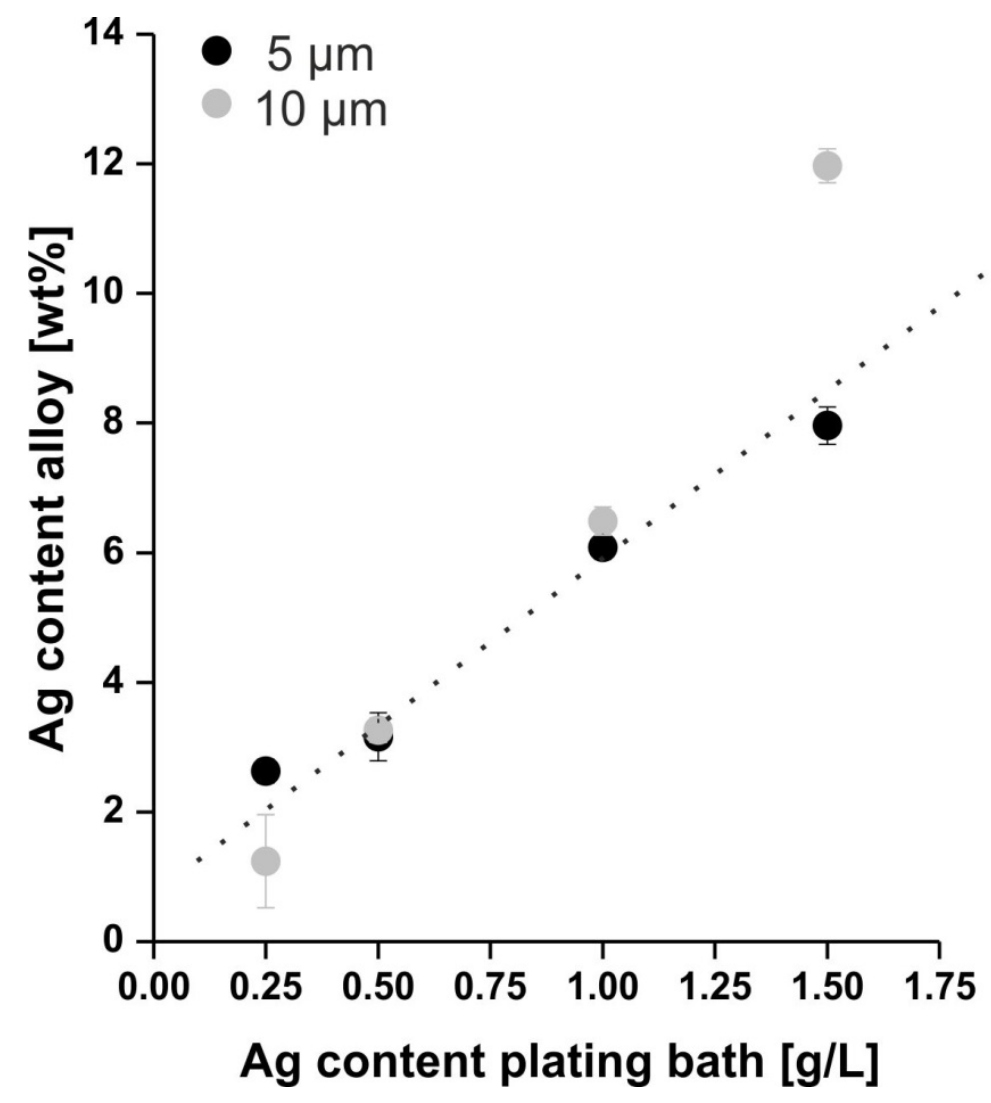

Figure S5. Dependence of the Ag alloy content determined by EDX on the Ag content in the plating bath for SnAg alloy films with different layer thicknesses. Electrodepositions were performed with Chemistry A and plating parameter set 1 given in Tables 1 and 2 of the manuscript, respectively. The dots resemble the mean Ag alloy content determined by EDX averaged over 3 positions on two independent film electrodeposits. Black dots refer to an electrodeposit layer thickness of $5 \mu \mathrm{m}$, grey dots to an electrodeposit layer thickness of $10 \mu \mathrm{m}$. It can be seen that the Ag alloy content is a linear function of the Ag content in the VMS, but does not change for rather thick electrodeposits between 5 or $10 \mu \mathrm{m}$, as already shown in Fig. S4. 


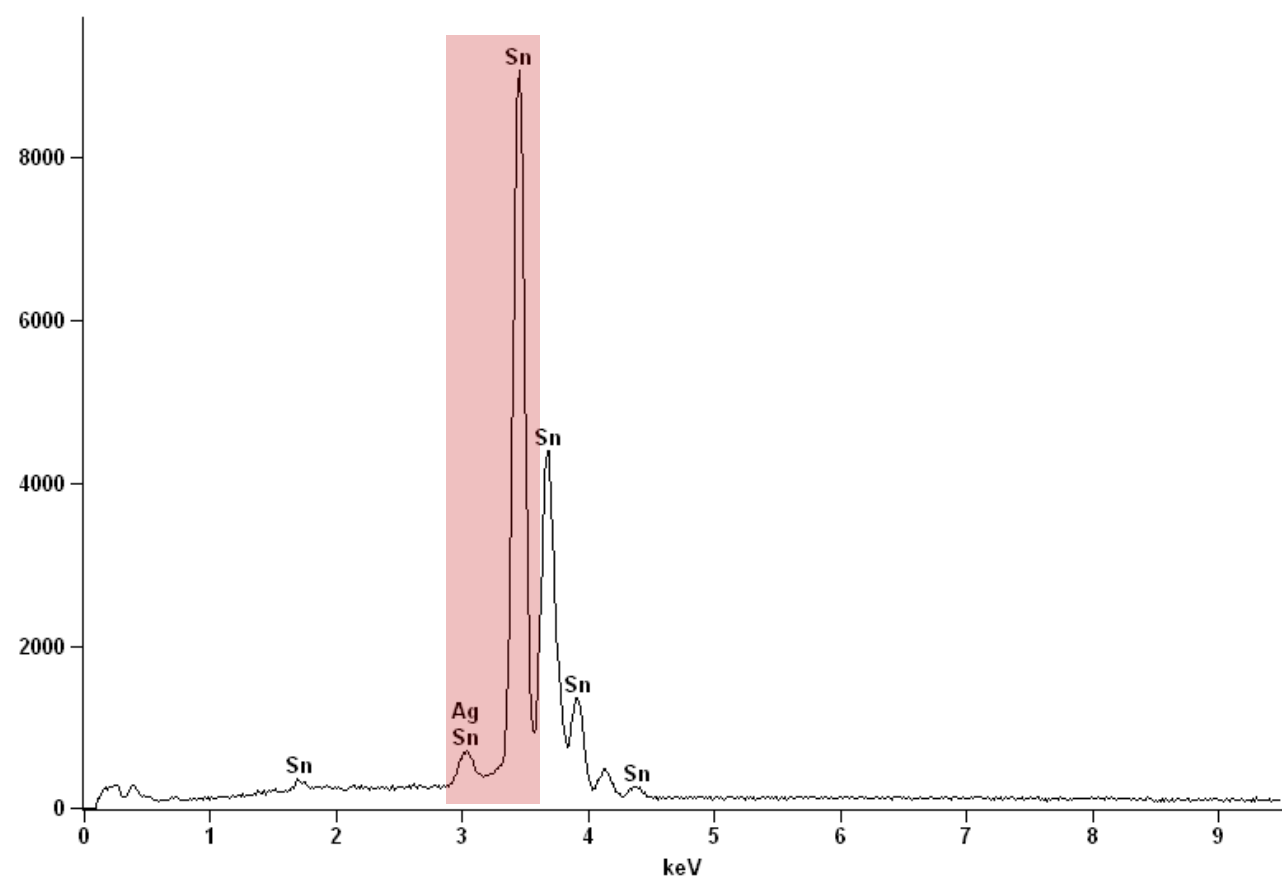

Figure S6. Representative EDX spectrum obtained for a SnAg electrodeposit layer of $5 \mu \mathrm{m}$ thickness plated with Chemistry B and plating parameters 1 (Tables 1 and 2 in the manuscript). The peaks for Sn and Ag are labelled. It can be seen that there is a substantial overlap of such peaks identified for Sn and Ag in the range of their L lines, indicated by the red rectangle. Exact values are given in the table below.

\begin{tabular}{|l|l|l|l|l|}
\hline & $\mathrm{L} \alpha_{1}$ & $\mathrm{~L} \alpha_{2}$ & $\mathrm{~L} \beta_{1}$ & $\mathrm{~L} \beta_{1}$ \\
\hline Ag & $2,984.31$ & $2,978.21$ & $3,150.94$ & $3,347.81$ \\
\hline Sn & $3,443.98$ & $3,435.42$ & $3,662.80$ & $3,904.86$ \\
\hline
\end{tabular}

[Data taken from http://xdb.lbl.gov/ on October 2, 2019]

The observed overlap systematically impairs the quantification of the Ag alloy fraction in SnAg by means of EDX. 

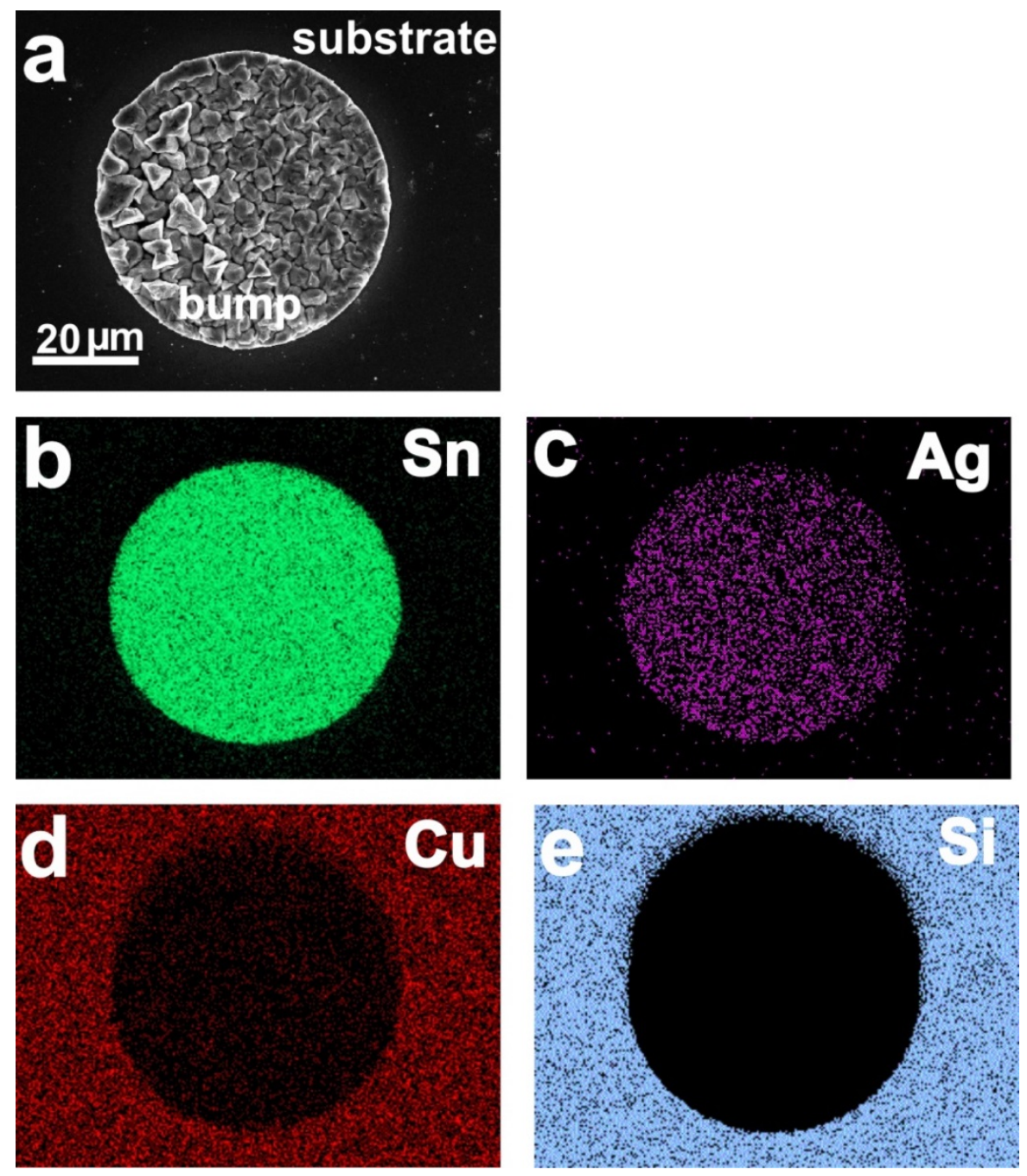

Figure S7. EDX mappings of Sn, Ag, Cu, and Si for an SnAg bump plated with Chemistry B and plating parameters 2 (Tables 1 and 2, see manuscript) on the Cu-seeded Si substrate. It can be seen in panel c) that the Ag distribution looks rather homogeneous. This is, however, an artefact due to the low spatial resolution of the EDX detector. Therefore, EDX, similar to ICP-MS, cannot provide spatially resolved chemical compositional data. 


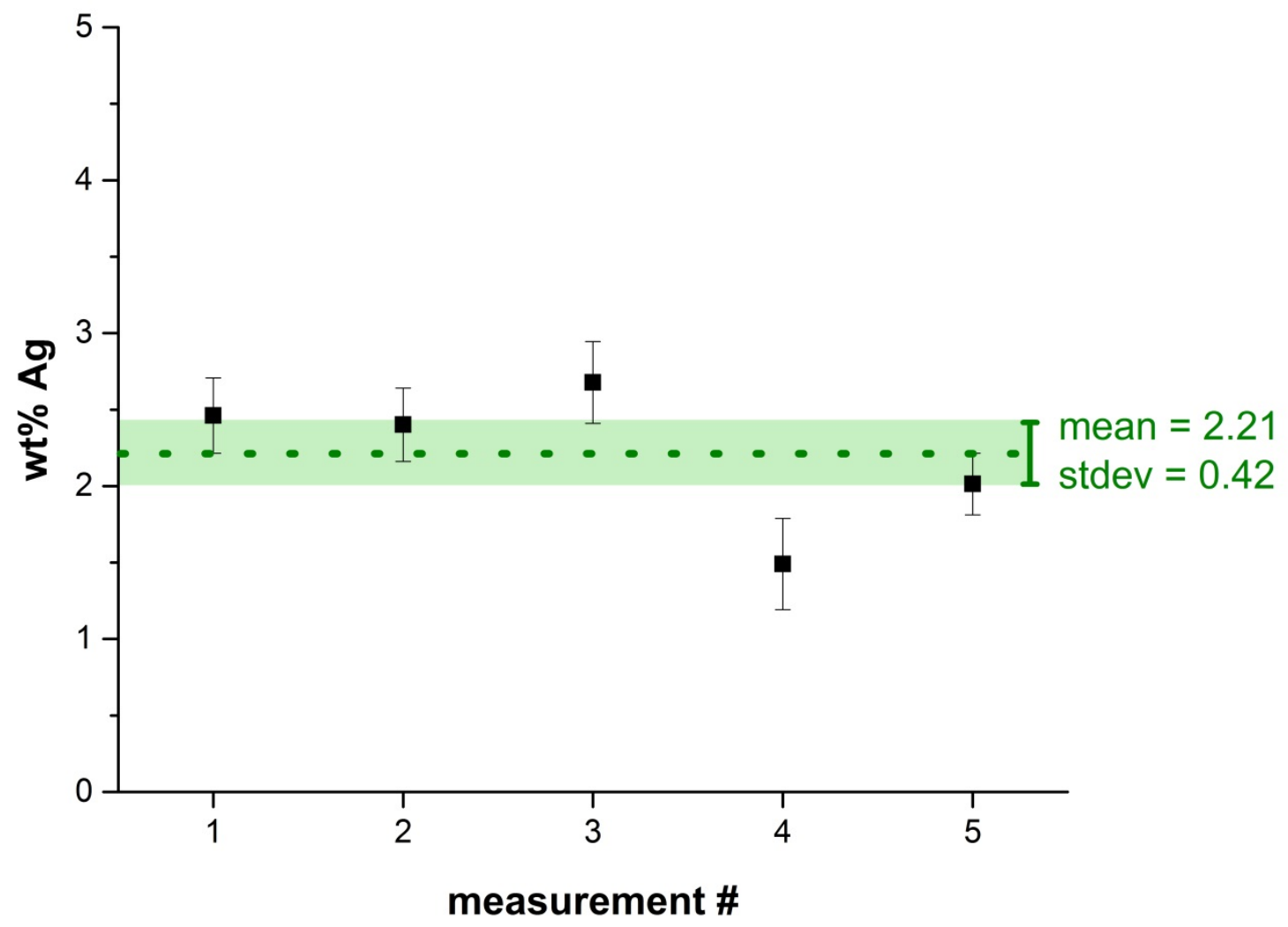

Figure S8. Overview of 5 LIMS measurements to determine the Ag weight fraction on 5 individual SnAg bumps. Measurements 1-3 were performed at $0.51 \mu \mathrm{J}$, measurements $4-5$ at $0.33 \mu \mathrm{J}$. All values were calibrated with the RSC determined for the respective laser pulse energy (see Fig. 3 in the main text). The center-to-center pitch of individual laser spots per layer was $4 \mu \mathrm{m}$ for measurement $1,6 \mu \mathrm{m}$ for measurement 2 , and $5 \mu \mathrm{m}$ for measurements $3-5$. There is no noticeable influence of the center-to-center pitch of laser spots per layer with respect to bulk (ablation of entire bump) compositional analysis which was performed here. Data were considered until the underlying Si substrate was reached. The mean value of these 5 measurements was used for comparison with ICP-MS and EDX measurements shown in Fig. 4 of the main text. 


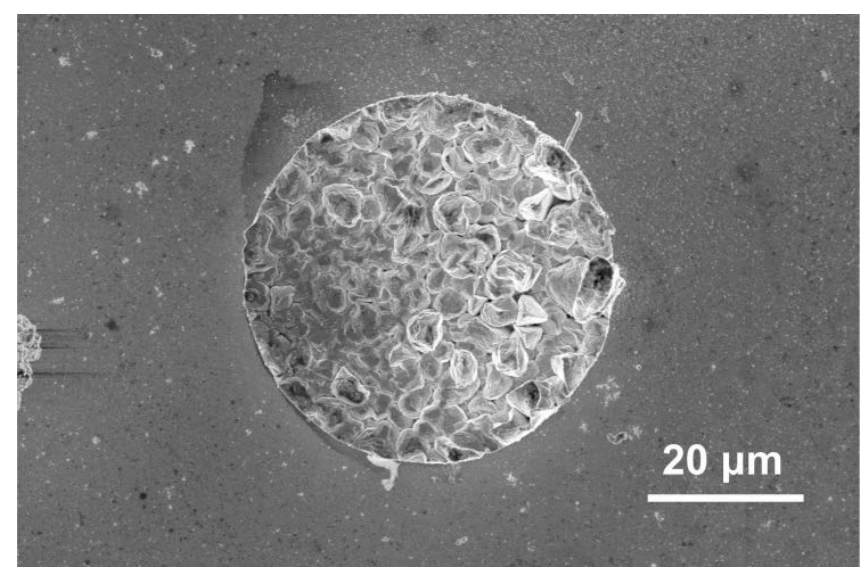

Figure S9. Top-down SEM image of the SnAg bump on which the LIMS depth profiling experiments shown in Figs. 5 and 6 were performed. No strong surface modifications are apparent, particularly no melting and recrystallization. 


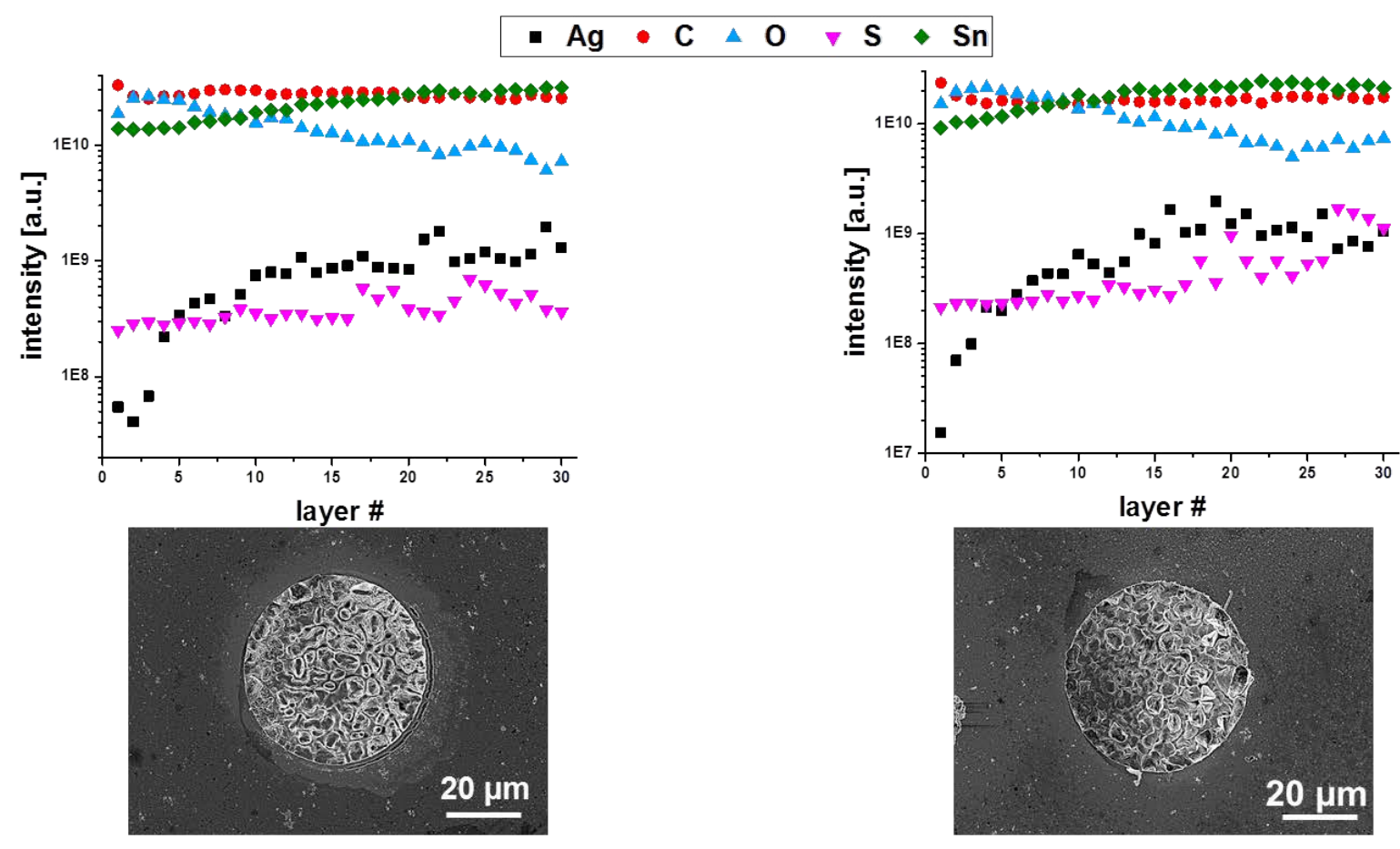

Figure S10. Results of two individual LIMS depth profiling experiments on distinct SnAg bumps performed at $0.33 \mu \mathrm{J}$ with 121 single laser spot positions per layer over 30 layers. The SEM images show the respective bumps after the experiment. Morphology and LIMS results are very closely comparable. 